\title{
Damage modeling of oxide/oxide ceramic matrix composites under cyclic loading conditions
}

\author{
Hui Liu ${ }^{\mathrm{a}}$, Huang Yuan ${ }^{\mathrm{a}, *}$, Zhengmao Yang ${ }^{\mathrm{b}}$ \\ ${ }^{\text {a }}$ School of Aerospace Engineering, Tsinghua University, Beijing, China \\ ${ }^{\mathrm{b}}$ Institute of Mechanics, China Academy of Science, Beijing, China
}

\section{A R T I C L E I N F O}

\section{Keywords:}

Oxide/oxide ceramic matrix composite

Continuum damage model

Elastic-plastic damage

Cyclic loading

\begin{abstract}
A B S T R A C T
Ceramic matrix composites exhibit optimal high temperature property and complex nonlinear behaviors including irreversible deformations and stiffness degradation under different mechanical loading conditions. In the present work, the damage evolution of the material under multi-axial loads considering effects of loading-unloading cyclic was studied and a novel continuum damage constitutive model was proposed. The material degradation was decomposed into monotonic damages and cyclic damages. The anisotropic hardening behavior of the material was considered by taking orientation dependence into account. Compared to the experimental results, the constitutive model could accurately predict the stress-strain response and stiffness degradations of the oxide/oxide ceramic matrix composites for monotonic loading and cyclic loading.
\end{abstract}

\section{Introduction}

Ceramic matrix composites (CMC) are widely considered as the important high-temperature materials for advanced turbine engines [1-3]. Compared to the other ceramic matrix composites, the oxide/ oxide ceramic matrix composite can withstand maximum temperature up to $1200{ }^{\circ} \mathrm{C}$ without additional environmental coatings and exhibit higher resistance to oxidation and hot corrosion [4-6]. Understanding the deformation and damage mechanisms is of essential importance for industrial application of the material.

Composites contain multiple components including matrix, fibers and interfaces, which lead to complex mechanical behaviors of the material [7-9]. Defects exist in the composites in forms of matrix cracks, fiber breakage and interface debonding etc. [9-12]. These microstructure damages accumulate and propagate during the loading process and cause complex macroscopic inelastic features into the material [13-15]. Because of coupling of damage and deformation, plasticity becomes inefficient to describe the macroscopic anisotropic behavior of the ceramic matrix composite, especially if the material undergoes cyclic loading.

To predict the nonlinear mechanical behavior of composites, various models were proposed for different scales, including micro/mesomechanical models [16-18] as well as macro-mechanical models [19-21]. Macro-mechanical models were widely used in industrial applications for simplicity and availability [16,22]. Most damage models for composites assumed that the loading-unloading process did not influence the mechanical behaviors of the composite, that is, the composite was dominated by the monotonic damage and not affected by fatigue. However, Guo et al. [23] found that the degradation of stiffness and strength of CMC under in-plane shear loading were significantly affected by repeated loading processes. Reversed loads induce additional damage into the material.

Hochard et al. [24,25] proposed an innovative damage model to describe effects of cyclic loading on the damage evolutions. The model decomposes the damage into static damage and fatigue damage. The evolution of fatigue damage was determined by both maximum stress and stress amplitude. Nevertheless this model only considered damage in the transverse direction and a simplified hypothesis was adopted so that the shear damage should be equivalent to the transverse damage, whereas in ceramic matrix composites the damage evolution in longitude direction, transverse direction and shear direction generally follow different rules. The model of Hochard cannot predict damage evolution under general loading conditions for its discontinuity.

Recently, plasticity coupling damage was adopted and effective stresses were used in the yield function to describe the irreversible deformation in composites. The plastic hardening of the composites was assumed to be orientation-independent in many published works, which did not agree with many experimental observations $[19,20,26,27]$. The kinematic hardening in combining with heterogeneous material behavior makes determine the backstress evolution

\footnotetext{
* Corresponding author.

E-mail address: yuan.huang@tsinghua.edu.cn (H. Yuan).
} 
difficult [28,29]. Additionally, composites reveal significant softening of the off-axis stress-strain relation and inelastic deformations are coupled with material degradation, which was not considered systematically [30,31]. Yang et al. [32-34] investigated thermo-mechanical damage in the oxide/oxide ceramic matrix composites under thermal shock condition and tries to introduce new damage variables. The models are limited in quantitative description of experiments and cannot be applied for computing CMC components. Inelastic behavior of the composites needs much more efforts in mechanical understanding and constitutive modeling.

In the present work an elastic-plastic damage constitutive model for cyclic loading configurations is proposed. The difference between the envelope curves of monotonic loading and cyclic loading is described by decomposing the damage into monotonic damage and cyclic damage. Two sets of anisotropic damage evolution equations were introduced to characterize the monotonic and cyclic damage separately. Based on the strain equivalence hypothesis, a novel plasticity model considering anisotropic hardening for various loading configurations is combined with the damage evolution. Experimental verification shows that the proposed constitutive model predicts mechanical behavior of the ceramic matrix composite with satisfactory accuracy.

\section{Experiments}

\subsection{Material and specimens}

The oxide/oxide ceramic matrix composite studied in the present work consists of the $\mathrm{Al}_{2} \mathrm{O}_{3}-\mathrm{SiO}_{2}-\mathrm{ZrO}_{2}$ matrix and the reinforced fiber Nextel $^{\mathrm{TM}} 610$ that exhibits advantageous specific mechanical properties including strength and stiffness. The material was fabricated via polymer infiltration and pyrolysis (PIP) process. The total porosity of the composite is $25 \mathrm{vol} \%$ to $35 \mathrm{vol} \%$, the density is $2.9 \mathrm{~g} / \mathrm{cm}^{3}$ and the fiber volume content of the composite is $35 \mathrm{vol} \%$ to $45 \mathrm{vol} \%$.

To study the influence of loading-unloading cycles on mechanical properties of the composite, three groups of experiments were carried out. The first set of Monotonic experiments ran under monotonic loading conditions. The experimental results served as the baseline in the present study. The second set of Monotonic-unloading experiments was referred to monotonic damage tests, in which four unloading loops were added before failure, to determine variations of the material's stiffness. Finally, the third set of Cyclic loading tests was conducted under cyclic loading conditions, in which the maximum displacement increases with small steps but with unloading to zero stress, so that the material is damaged by cyclic loads. The significant difference of the test is in increasing maximum displacement, while a fatigue test is run under a given maximum displacement or maximum strain. Due to increasing maximum displacement, the material fails after few loading cycles. From viewpoint of constitutive modeling, the cyclic constitutive model verified in the present work should be able to describe fatigue damage.

Specimens investigated in the present work were machined by water-jet. The dog-bone shaped specimen shown in Fig. 1 (a) was used for the tensile tests. The material property was identified from uniaxial tensile tests with different off-axis angles of $0^{\circ}, 30^{\circ}, 45^{\circ}, 90^{\circ}$, respectively. The strain rosette was installed in the specimen to determine the strain state and to calculate the elastic modulus variation in the corresponding principal material directions. The Iosipescu shear tests illustrated in Fig. 1(b) were conducted for shear strength.

All monotonic tensile tests were run under displacement control, with a constant grip velocity of $0.001 \mathrm{~mm} / \mathrm{s}$, while the loading-unloading tests were quicker with $0.003 \mathrm{~mm} / \mathrm{s}$. The unloading was controlled by given force and stopped as the force was released. All tests were conducted in an MTS hydraulic-server universal test machine.

\subsection{Experimental results}

In continuum damage mechanics the damage is usually represented
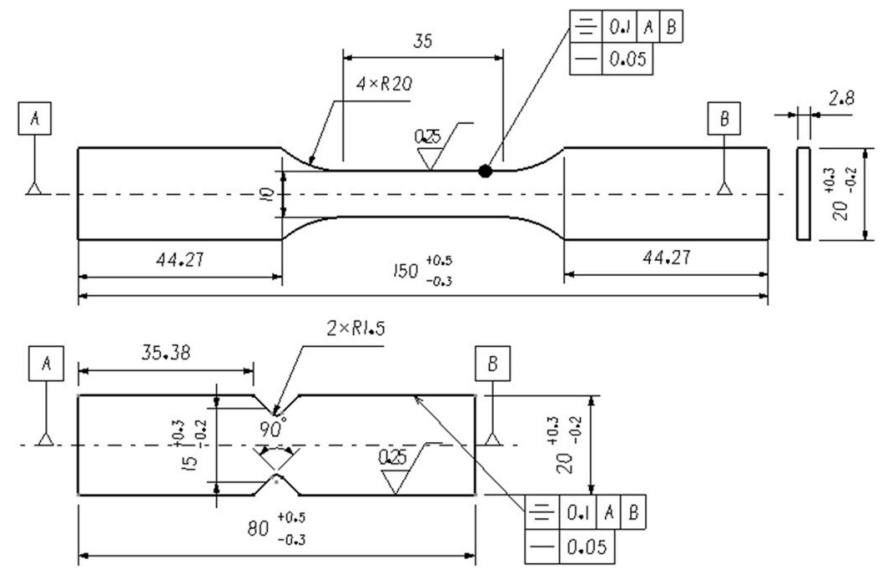

Fig. 1. Specimen geometry investigated in the present work. (a) The uniaxial tensile specimen; (b) the Iosipescu shear specimen.

by reduction of the elastic modulus [35]. The degradation of the material property can be identified from tensile tests with unloading [36-38]. The stress-strain curves from monotonic tests and monotonicunloading tests are plotted in Fig. 2 for different loading configurations. The stress-strain curves in both $30^{\circ}$ and $45^{\circ}$ off-axis directions are plotted together with results from Iosipescu shear tests. In the figure the solid lines denote the monotonic loading results, whereas the dashed curves stand for tensile tests with unloading. The unloads were induced to determine variations of elastic modulus. It is confirmed that the envelop of the loading-unloading curves approach the monotonic curves, which implies negligible effects from the four unloads the material behavior. The unloads did not induce additional damage into the specimens under both tension and shear conditions. The obtained elastic modulus can represent material degradation under the monotonic loading.

The situation changes with increasing loading cycles, as one may expect from fatigue. Fig. 3 illustrates cyclic stress-strain curves in different off-axis directions with loading-unloading cycles. The tension is controlled by increasing displacement and unloading is controlled by stress release. For $0^{\circ}$ and $90^{\circ}$ cyclic loading tests, only the initial cycles and the last cycles are plotted in Fig. 3(a) and (d), respectively. Both $0^{\circ}$ and $90^{\circ}$ directions are nearly elastic, however, slight irreversible strains and stiffness degradations can be observed during the tests. Cyclic loading generates additional damage into the material.

Both $30^{\circ}$ and $45^{\circ}$ off-axis tests demonstrate significantly larger plastic deformations and the material behaves ductile in the off-axis direction. The cyclic loading diminishes loading capability of the material. Obviously, the material was damaged by the cyclic loading and the influence of loading-unloading cycles on the mechanical response becomes significant.

To quantitatively describe the degradation of the oxide/oxide ceramic matrix composite, damage can be defined based reduction of the elastic stiffness in different directions for the orthogonal material, as

$d_{1}=1-E_{1} / E_{1}^{0}$,

$d_{2}=1-E_{2} / E_{2}^{0}$,

$d_{6}=1-G_{12} / G_{12}^{0}$,

in the principal material coordinates. $E_{1}$ and $E_{2}$ are the current elastic moduli in the both fibre reinforced directions, whereas $E_{1}^{0}$ and $E_{2}^{0}$ represent their initial values. $d_{1}, d_{2}$ and $d_{6}$ stand for material degradations in the corresponding material directions, respectively.

From the experiments presented previously, the damage indicators $d_{i}$ can be evaluated directly. For the cyclic loading the hysteresis loops are not considered in the calculation. The current elastic modulus is taken from the slope of the corner connect line. The damage evolution in three series of tests is summarized in Figs. 4 and 5. 


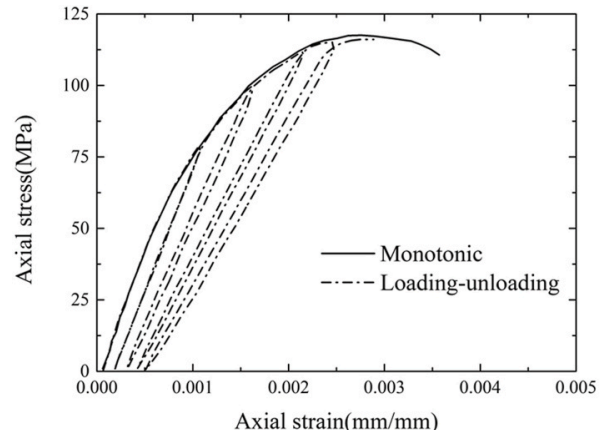

(a)

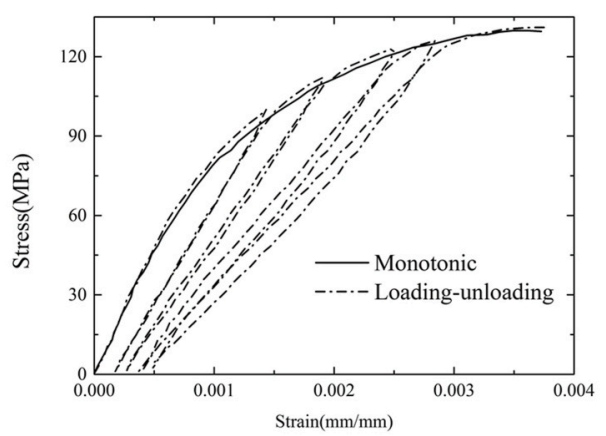

(b)

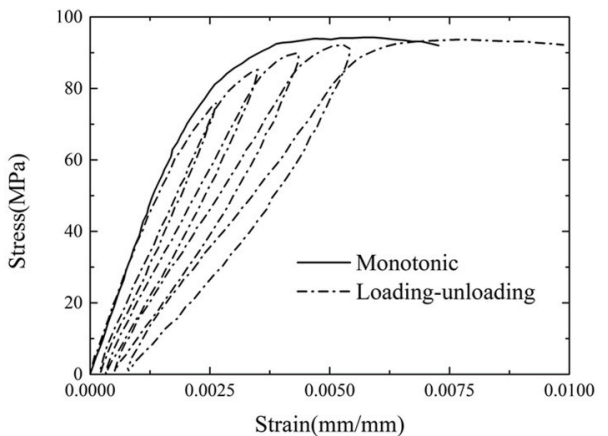

(c)

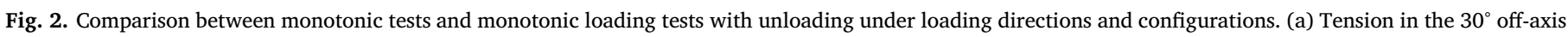
direction. (b) Tension in the $45^{\circ}$ off-axis direction. (c) Iosipescu shear tests.

As discussed, the four unloads in the monotonic tests did not affect the damage evolution in the material. Fig. 4 illustrates evolutions of damage in both $30^{\circ}$ and $45^{\circ}$ off-axis specimens as a function of the applied load. As observed, the material did not show degradation in $0^{\circ}$ and $90^{\circ}$ directions before broken. While the shear damage from the Iosipescu test stands for the shearing in the material coordinates, the shear damage was additionally identified from both off-axis tensile specimens based in strain measurements. Additionally, the shear modulus of the material can be evaluated from a $45^{\circ}$ off-axis tensile test, in accordance with the ASTM standard D 3518 [39]. The axial strain $\varepsilon_{x}$

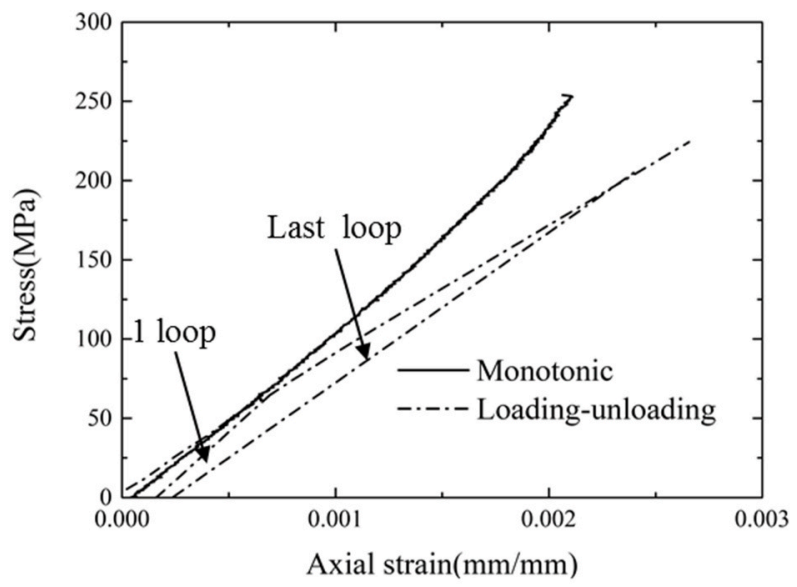

(a)

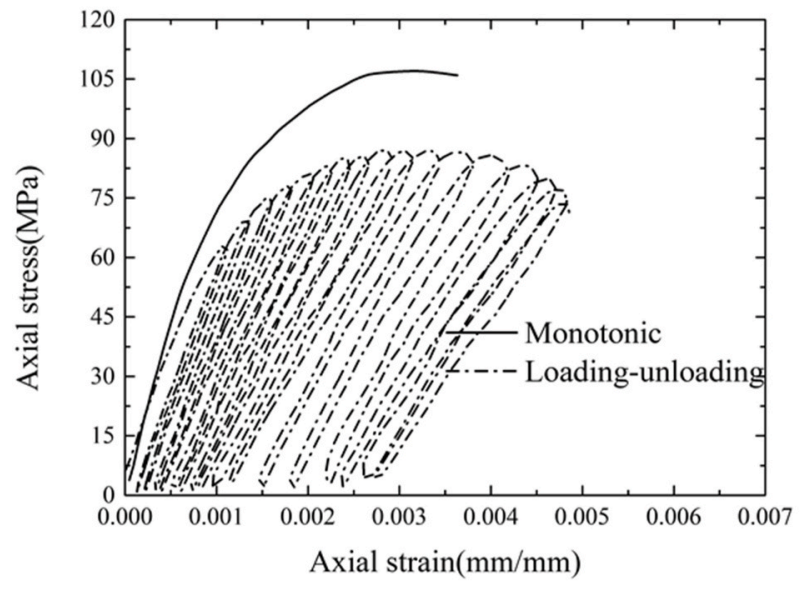

(c) and the transverse strain $\varepsilon_{y}$ are measured by a strain gage rosette. The shear modulus $G_{12}$ can be obtained from

$G_{12}=\frac{\sigma_{x}}{2\left(\varepsilon_{x}-\varepsilon_{y}\right)}$,

where $\sigma_{x}$ notes the tensile stress, $\sigma_{x}=F / A$, where $A$ is the cross-sectional area in tensile direction of the specimen. Reduction of the shear modulus represents the shear damage in the off-axis specimens. The results show that the damage from tension is affected by the off-axis angle slightly, whereas the shear damage in the material coordinate

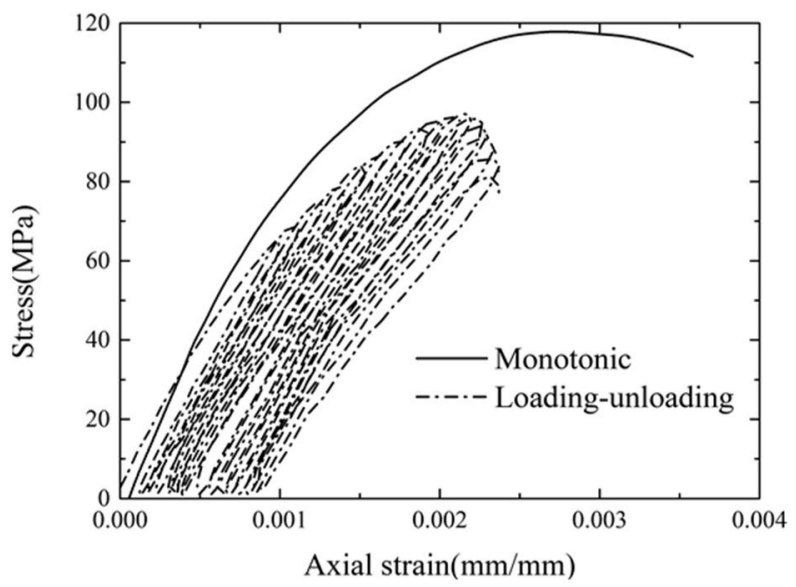

(b)

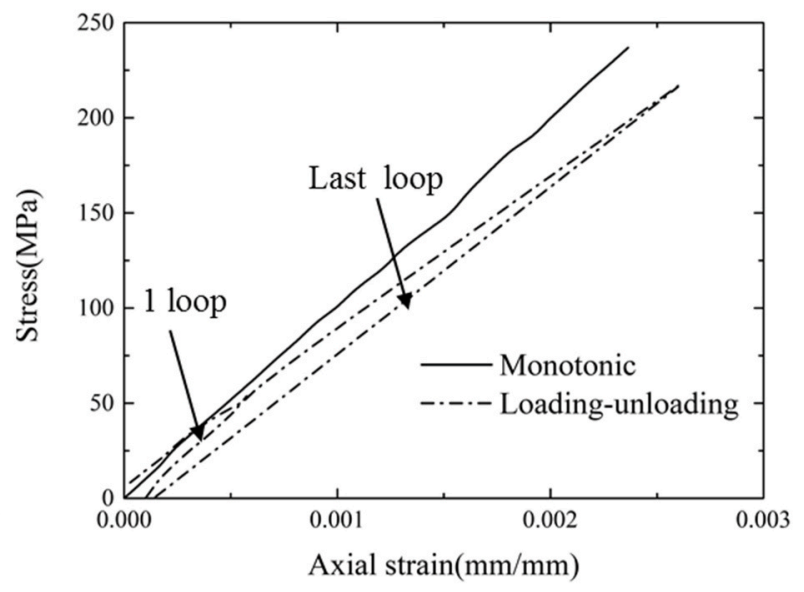

(d)

Fig. 3. The stress-strain curves of monotonic and cyclic damage experiments in different directions. (a) $0^{\circ}$, (b) $30^{\circ}$, (c) $45^{\circ}$, (d) $90^{\circ}$. 


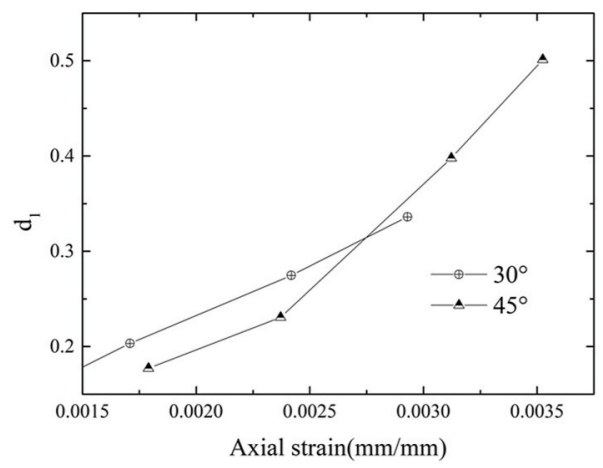

(a)

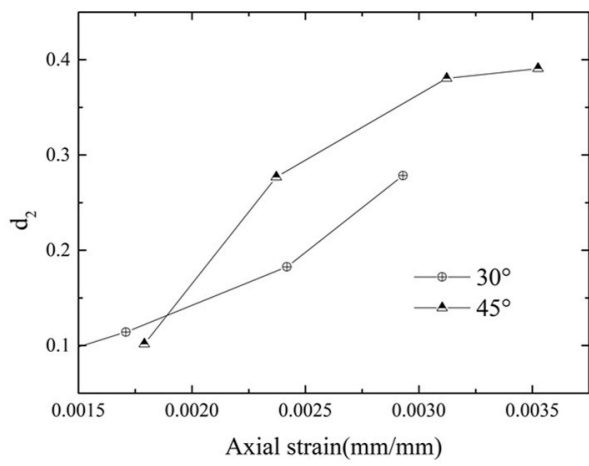

(b)

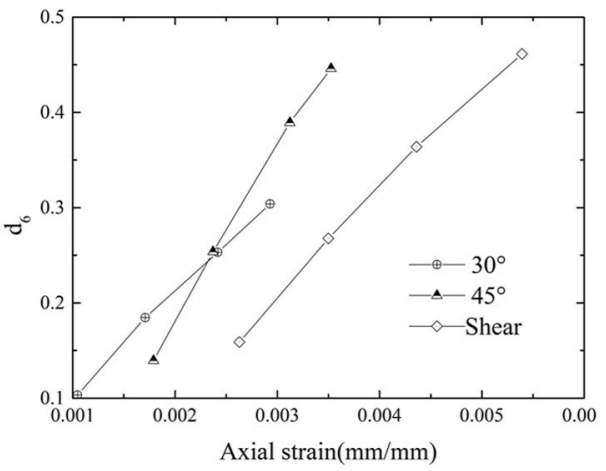

(c)

Fig. 4. Experimental results of damage evolutions under monotonic loading conditions. (a) $d_{1}$. (b) $d_{2}$. (c). $d_{6}$

develops significantly slower than the others.

The damage under cyclic loading conditions develops differently from the monotonic damage, as shown in Fig. 5. Both off-axis angles $30^{\circ}$ and $45^{\circ}$ reveal very different evolution trends. There is little damage in the fiber directions in the on-axis $\left(0^{\circ} / 90^{\circ}\right)$ monotonic loading specimens, however, under cyclic loading conditions the damage in the onaxis direction is obvious. But the final failure occurs at a low damage level, as shown in Fig. 5. Whereas damage in $30^{\circ}$ increases rapidly and results in failure within small strains, the specimen of the $45^{\circ}$ behaves even more ductile than that under monotonic loading condition, which can be confirmed from Fig. 3(b). Only for small strains both off-axis specimens share the similar damage evolution. The shear damage was calculated from the tension and given the similar features as the tensile damage.

It is known that the CMC is rather brittle. However, Figs. 2 and 3 reveal significant inelastic deformations, especially in the off-axis specimens. From discussions above, the inelastic strain consists of plastic strain and damage-induced strain, $\varepsilon^{\mathrm{ie}}=\varepsilon^{\mathrm{p}}+\varepsilon^{\mathrm{d}}$. In Fig. 6, plastic strains in different specimens are summarized under both monotonic and cyclic loading conditions, where the plastic strain is defined as $\varepsilon^{p}=\varepsilon-\sigma /(E(1-d))$. Such decomposition implies that the damageinduced inelastic strain is included in the elastic strain.

As expected, the plastic strain in the on-axis direction is in the level of $0.01 \%$ for both monotonic and cyclic loads. For off-axis loading the material displays significant different plastic deformation capacity. Whereas the monotonic loading tests reached a plastic strain larger than $0.1 \%$ for the $30^{\circ}$ and $0.2 \%$ for $45^{\circ}$ specimen, the accumulative plastic strain under cyclic loading is generally much smaller. Although the stress-strain curves display significant inelastic deformations, the plastic strain is small in comparing with the damage-induced inelastic behavior.

\section{Cyclic elastic-plastic damage constitutive model}

\subsection{The framework of damage mechanics}

By neglecting the internal friction, the strain increments in the composite can be divided into elastic strain increment and plastic strain increment, as

$\mathrm{d} \varepsilon=\mathrm{d} \varepsilon^{\mathrm{e}}+\mathrm{d} \varepsilon^{\mathrm{p}}$.

Above the elastic strain increment is related to stress via the Hooke's law,

$\mathrm{d} \varepsilon^{\mathrm{e}}=\mathbf{C}: \mathrm{d} \tilde{\sigma}$,

where $\tilde{\sigma}$ is the effective stress tensor of the damaged material. Based on the strain equivalence hypothesis $[35,40]$, the effective stress tensor is related to the nominal stress as

$\tilde{\sigma}=\mathbf{M}: \sigma$

with the inverse damage tensor (40)

$\mathbf{M}=\left[\begin{array}{ccc}\frac{1}{1-d_{1}} & 0 & 0 \\ 0 & \frac{1}{1-d_{2}} & 0 \\ 0 & 0 & \frac{1}{1-d_{6}}\end{array}\right]$

for the plane stress state. The effective stress tensor for the plane stress state can be expressed in a vector form as

$\tilde{\sigma}=\left[\begin{array}{l}\tilde{\sigma}_{11} \\ \tilde{\sigma}_{22} \\ \tilde{\sigma}_{12}\end{array}\right]=\left[\begin{array}{c}\frac{\sigma_{11}}{1-d_{1}} \\ \frac{\sigma_{22}}{1-d_{2}} \\ \frac{\sigma_{12}}{1-d_{6}}\end{array}\right]$.

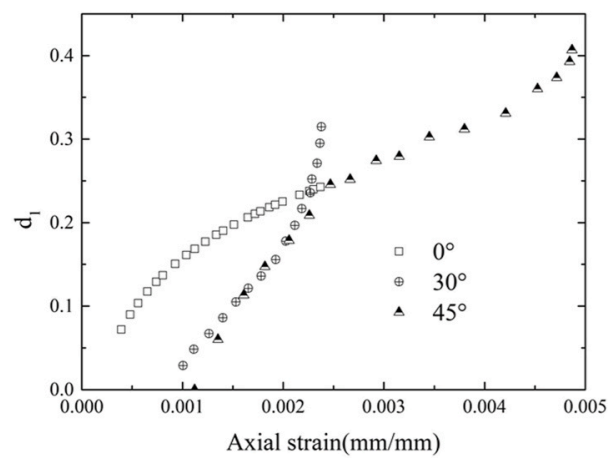

(a)

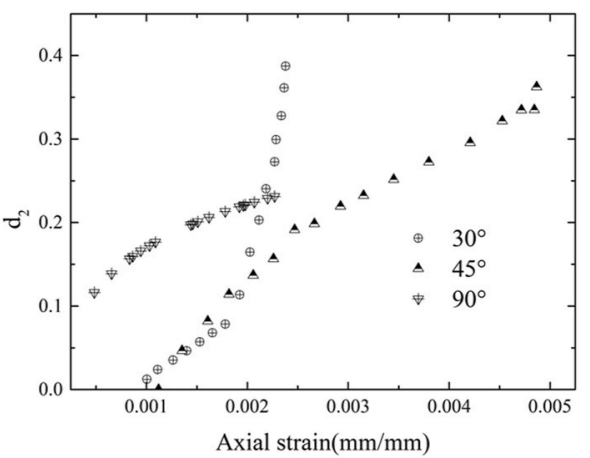

(b)

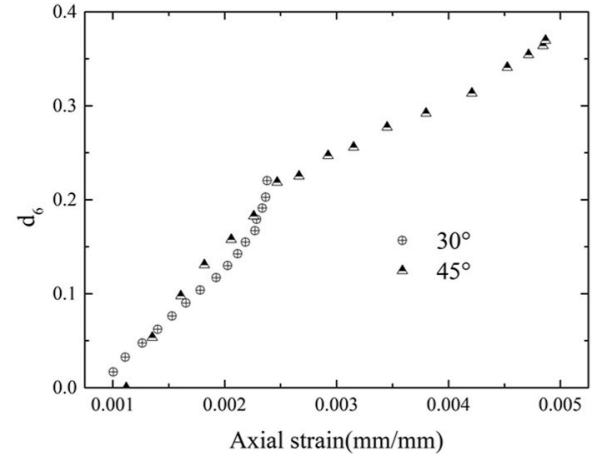

(c)

Fig. 5. Experimental results of the damage evolution under cyclic loading conditions. (a) $d_{1}$. (b) $d_{2}$. (c). $d_{6}$ 


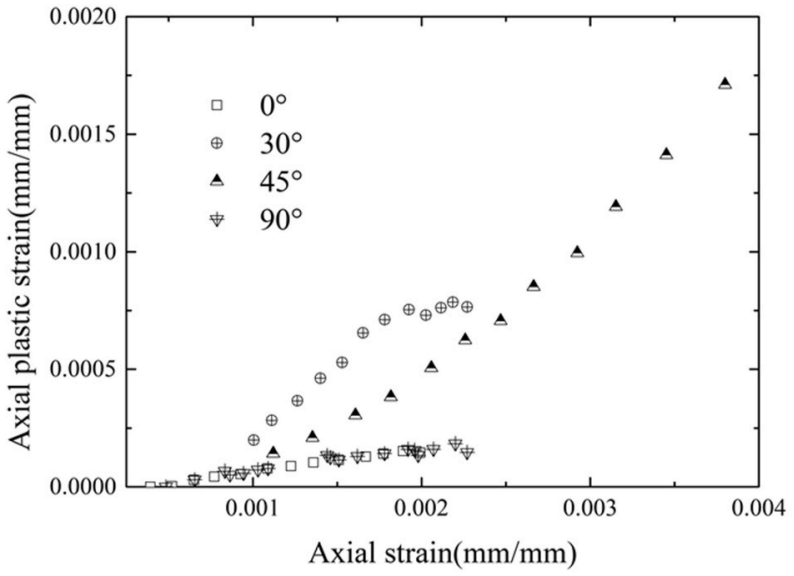

(a)

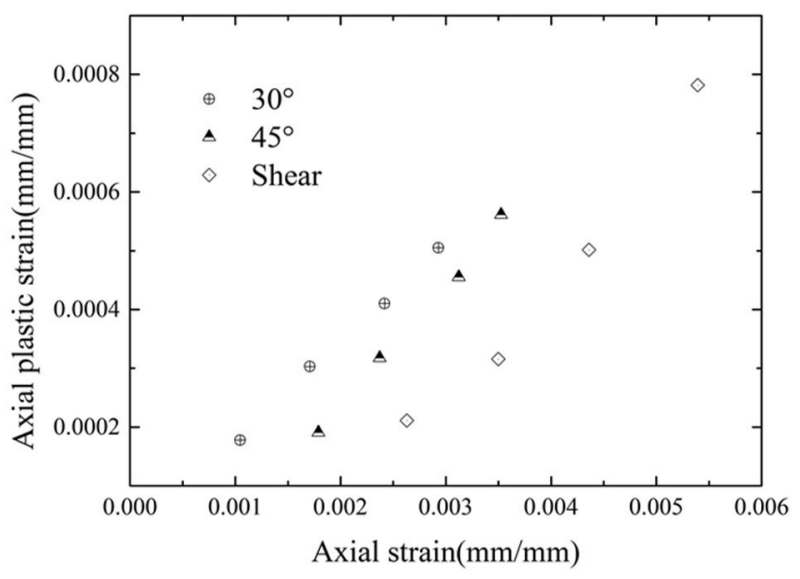

(b)

Fig. 6. Plastic strain evolutions for monotonic and cyclic loading conditions. (a) Monotonic loading conditions. (b) Cyclic loading conditions.

All other stresses components are zero under plane stress conditions. C is the initial compliance tensor of the composite and can be expressed as

$\mathbf{C}=\left[\begin{array}{ccc}\frac{1}{E_{11}} & -\frac{\nu_{21}}{E_{11}} & 0 \\ -\frac{\nu_{21}}{E_{11}} & \frac{1}{E_{22}} & 0 \\ 0 & 0 & \frac{1}{G_{12}}\end{array}\right]$,

where $E_{11}$ and $E_{22}$ are elastic moduli in both fibre directions. $v_{21}$ represents Poisson's ratio. $G_{12}$ stands for shear modulus. The composite is orthogonal anisotropic with four independent elastic material constants for the plane problem. The Hooke's law can be further expressed as

$\varepsilon^{\mathrm{e}}=\mathbf{C}: \tilde{\sigma}=\tilde{\mathbf{C}}: \sigma$,

where $\tilde{\mathbf{C}}=\mathbf{C} \cdot \mathbf{M}$ is the compliance matrix of the damaged material. Note that $\tilde{\mathbf{C}}$ is symmetric matrix generally. For simplicity, only the orthotropic damage is considered in the present work, that is, the material is assumed to maintain orthotropic in the loading process. $\tilde{\mathbf{C}}$ is expressed as

$\tilde{\mathbf{C}}=\left[\begin{array}{ccc}\frac{1}{E_{11}\left(1-d_{1}\right)} & -\frac{\nu_{21}}{E_{11}} & 0 \\ -\frac{\nu_{21}}{E_{11}} & \frac{1}{E_{22}\left(1-d_{2}\right)} & 0 \\ 0 & 0 & \frac{1}{G_{12}\left(1-d_{6}\right)}\end{array}\right]$

In the present material both elastic moduli $E_{11}$ and $E_{22}$ are in the same range, so that, such a simplification does not induce substantial deviations.

If the material is considered to be elastic-plastic, the plastic strain has to be derived based on a potential function. A novel elastic-plastic damage combined constitutive model was proposed to simulate the nonlinear mechanical response in general stress states for monotonic loading and cyclic loading-unloading conditions [38,41]. In the proposed model, the continuum damage mechanics theory was used to describe the stiffness degradation of the matrix material and the plasticity was utilized to determine the plastic strain. The thermodynamic potential $\psi$ which describes the material state can be expressed as

$\psi=\psi_{\mathrm{e}}(\sigma, \mathbf{D})+\psi_{\mathrm{p}}(p, \mathbf{D})$,

where $\psi_{\mathrm{e}}$ and $\psi_{\mathrm{p}}$ are connected to the elastic and plastic behaviors coupled with damage, respectively. $p$ is the equivalent plastic strain corresponding to plastic flow which can be obtained by plasticity theories.

From the experiments, the damage evolutions in monotonic loading tests and cyclic loading-unloading tests are different. In fact, the cyclic damage is induced after the monotonic loading, that is, the damage can be decomposed into monotonic damage and cyclic damage [38], as

$\dot{d}_{i}=\dot{d}_{i}^{\mathrm{m}}+\dot{d}_{i}^{\mathrm{c}}$

with ( $i=1,2,6)$, respectively. $d_{i}$ is the total damage component in the principal direction of the material, $d_{i}^{\mathrm{m}}$ describes the monotonic damage and $d_{i}^{\mathrm{c}}$ represents the cyclic damage which only occurs during cyclic loading. $(\dot{x})$ is the rate of $x$.

\subsection{Plasticity model}

In the frame of plasticity, the plastic deformation for the matrix is characterized by

$f(\tilde{\sigma})=\kappa\left(\bar{\varepsilon}^{\mathrm{p}}\right)$,

where $f(\tilde{\sigma})$ is the potential function defined in the effective stress space for the plastic behavior and $\kappa\left(\bar{\varepsilon}^{\mathrm{p}}\right)$ represents the current yield stress of the material depending on plastic strain [41]. The effective stress $\tilde{\sigma}$ was defined in Eq. (7) and applied for the damage model.

The Hill-type yield function is widely used to describe the yield behaviors of different materials, however, limited applicable for the composites. Sun and Chen [26] proposed a yield function for unidirectional composite, which shows significant orientation-dependence in plastic behavior, as

$f\left(\sigma_{i j}\right)=\frac{1}{2} \sigma_{22}^{2}+a_{66} \sigma_{12}^{2}$.

The model above is suitable for fibre-reinforced material, but not for woven composites. Liu et al. [41] generalized the yield function for woven composites and considered the off-axis material behavior. For the damaged material, the stress in the function should be replaced by the effective stress, as

$f\left(\tilde{\sigma}_{i j}\right)=\sqrt{F\left(\tilde{\sigma}_{11}+\mu \tilde{\sigma}_{22}\right)^{2}+2 \tilde{\sigma}_{12}^{2}}$

with $F$ and $\mu$ as model parameters. Using the associated flow rule, the effective equivalent stress describing the stress state is defined as

$\widetilde{\sigma}=f\left(\tilde{\sigma}_{i j}\right)$

and the plastic strain increment can be obtained from the flow rule (35),

$\mathrm{d} \varepsilon_{i j}^{p}=\mathrm{d} \lambda \frac{\partial f}{\partial \sigma_{i j}}$,

where $d \lambda$ is the plastic multiplier to be determined in the stress integration. 
According to plasticity theory, the increment of the plastic work can be written as

$\mathrm{d} W_{\mathrm{p}}=\tilde{\sigma}_{i j} \mathrm{~d} \varepsilon_{i j}^{\mathrm{p}}=\tilde{\sigma} \mathrm{d} \bar{\varepsilon}^{\mathrm{p}}$

with $d \bar{\varepsilon}^{p}$ as the incremental equivalent plastic strain. Substituting the incremental plastic strain into Eq. (18) follows

$\mathrm{d} \lambda=\mathrm{d} \bar{\varepsilon}^{\mathrm{p}}$.

As soon as the incremental equivalent plastic strain is found, the hardening rule considering the softening behavior of the ceramic matrix composite can be expressed as

$\kappa\left(\bar{\varepsilon}_{\mathrm{p}}\right)=A\left(\bar{\varepsilon}_{\mathrm{p}}\right)^{n} \exp \left(-m\left\langle\bar{\varepsilon}_{\mathrm{p}}-\varepsilon_{\mathrm{p}}^{0}\right\rangle^{t}\right)+\sigma^{0}$.

Above $\sigma^{0}$ represents the equivalent stress in the initial yield point and should be independent of the loading conditions. The exponents $n$ and $t$ describe effects of plastic strain hardening. $A$ and $m$ are functions of loading orientation and have to be determined from off-axis tensions [41].

To introduce anisotropy into the hardening rule, Liu et al. [41] introduced a dimensionless parameter characterizing the loading condition, as

$\omega=\frac{\left|\sigma_{12}\right|}{\bar{\sigma}}$.

The orientation-dependence of the plastic strain hardening is related to $\omega(41)$.

\subsubsection{Monotonic damage evolution}

The elastic part of the thermodynamic potential is defined to be the complementary elastic strain energy density. $\psi_{e}$ for orthotropic materials in the plane stress state can be expressed as

$\psi_{e}=\frac{1}{2}\left[\frac{\sigma_{11}^{2}}{E_{11}\left(1-d_{1}\right)}-\frac{2 \nu_{12} \sigma_{11} \sigma_{22}}{E_{11}}+\frac{\sigma_{22}^{2}}{E_{22}\left(1-d_{2}\right)}+\frac{\sigma_{12}^{2}}{G_{12}\left(1-d_{6}\right)}\right]$.

According to continuum damage mechanics, the elastic strain $\varepsilon_{i j}^{e}$ and the strain energy release rate as the damage driving force, $Y_{i}(i=1,2,6)$, associated with state variables, $\sigma_{i j}$ and $d_{i}$ can be obtained from

$\varepsilon_{i j}^{\mathrm{e}}=\rho \frac{\partial \psi_{e}}{\partial \sigma_{i j}}, \quad Y_{i}=\frac{\partial \psi_{e}}{\partial d_{i}}$.

The energy release rate $Y_{i}$ is the generalized thermodynamic force conjugated with the damage $d_{i}$. Substituting Eq. (22) into Eq. (23), the energy release rate is expressed as

$Y_{1}=\frac{\sigma_{11}^{2}}{2 E_{11}\left(1-d_{1}\right)^{2}}$,
$Y_{2}=\frac{\sigma_{22}^{2}}{2 E_{22}\left(1-d_{2}\right)^{2}}$,
$Y_{6}=\frac{\sigma_{12}^{2}}{2 G_{12}\left(1-d_{6}\right)^{2}}$.

To ensure the thermodynamic consistency, the increment of the damage variable in the whole loading process has to satisfy

$\dot{d}_{i} \geq 0$.

In the framework of the proposed damage model, the evolution law of the damage is established on the space of associated thermodynamic forces, $Y_{i}$. The monotonic damage can be expressed by the strain energy uniquely, so that the monotonic damage rate can be expressed as a unique function of $Y_{i}^{*}$, as

$\dot{d}_{i}^{\mathrm{m}}=\frac{\gamma_{i}}{t_{i}}\left\langle Y_{i}^{*}-Y_{i}^{0}\right\rangle^{t_{i}-1} \dot{Y}_{i}^{*}$,

where $\langle\cdot\rangle$ denotes the MacAuley brackets defined as $\langle x\rangle=(x+|x|) / 2$. The index $i=1,2,6$ represents the active stress component for the damage evolution. $Y_{i}^{0}$ is the threshold value for strain energy in damage nucleation. $\gamma$ and $t$ are model parameters. For a monotonic loading process the damage evolution can be integrated, as

$d_{i}^{\mathrm{m}}=\gamma_{i}\left(Y_{i}^{*}-Y_{i}^{0}\right)^{t_{i}}$.

The equivalent energy release rate $Y_{i}^{*}$ defined as

$\left\{\begin{array}{l}Y_{1}^{*}=a_{11} Y_{1}+a_{12} Y_{2}+a_{16} Y_{6} \\ Y_{2}^{*}=a_{21} Y_{1}+a_{22} Y_{2}+a_{26} Y_{6}, \\ Y_{6}^{*}=a_{61} Y_{1}+a_{62} Y_{2}+a_{66} Y_{6}\end{array}\right.$

to describe interactions between different stress components. Above $a_{i j}$ describes coupling effects of different thermodynamic forces in damage evolution and is to be determined based on experimental data. In the present damage model it is assumed that the monotonic damage is induced by stress, so that effects of the plastic strain are neglected.

\subsubsection{Cyclic damage evolution}

As the additional damage is induced by cyclic loads, the damage evolution law for the cyclic damage should reflect cycling stress/strain and loading paths.

In the present study, it is assumed that the cyclic damage only occur in the reloading process. when the effective equivalent stress in the current state during the reloading process is lower than the maximum effective equivalent stress in the whole loading history, defined as $\widetilde{\sigma}_{\max }=\max (\widetilde{\sigma}, t)$.

According to the Hochard's model $(24 ; 25)$, the cyclic damage is considered to be related to both maximum and amplitude of the thermodynamic force in each loading cycle. However in the Hochard's model, only damage in the transverse direction was considered and the shear damage was assumed to be equal to the transverse damage. Furthermore, Hochard et al. $(24 ; 25)$ assumed constant loading amplitude, so that the damage evolution equation cannot be applied to cyclic loading with variable amplitude. Their model can only be used to predict fatigue life under constant loading amplitude.

According to investigations in $(42 ; 38)$, the fatigue damage can be assumed to be driven by the plastic strain energy increment. In multiaxial fatigue assessment it is common to take the plastic strain energy density as failure criterion. From damage mechanics, it is known that the damage growth is related to the plastic strain as well as the damage state. With increasing plastic strain the material damage accelerates. Following suggestion from (38), the damage evolution equation can be written in the form as

$\dot{d}_{i}^{c}=\zeta_{i} \widetilde{\sigma}_{\max } \frac{d_{i}^{\alpha_{i}}}{\left(\bar{\varepsilon}^{p}\right)^{\beta_{i}}} \dot{W}$

where $i=1,2,6$ denotes the different damage components. $\bar{\varepsilon}^{p}$ is the accumulative equivalent plastic strain. $\zeta, \alpha$ and $\beta$ are model parameters.

Coupling of different loads is considered through the plastic strain as well as the plastic strain energy rate, as suggested in Refs. [38,42]. Due to the heterogeneity in the different directions, the model parameters can be determined from tests in different orientations.

\subsection{Identification of model parameters}

\subsubsection{Determination of the yield function}

The conventional method to calculate the parameters in the yield surface function was submitting the initial yield stress in various loading conditions into the expression of yield surface and solving these equations. However it is difficult to determine the initial yield point for the damaged material. According to Eq. (17), the increment of plastic strain for the damaged material could be written as 


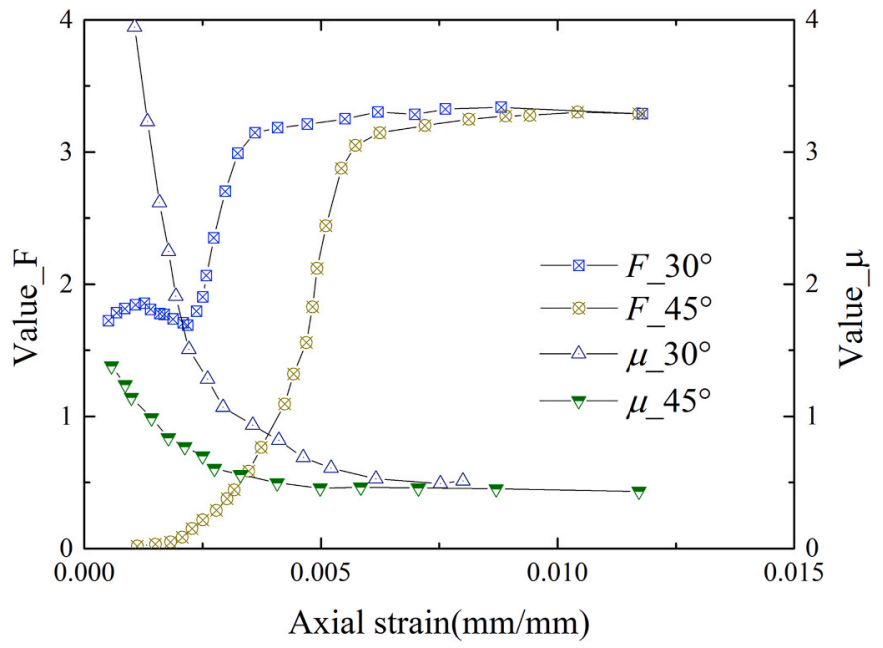

Fig. 7. Variations of parameters $F$ and $\mu$ in different tensile tests.

$\left\{\begin{array}{l}\mathrm{d} \varepsilon_{11}^{\mathrm{p}}=\mathrm{d} \lambda \frac{\partial f}{\partial \sigma_{11}}=\frac{F\left(\tilde{\sigma}_{11}+\mu \tilde{\sigma}_{22}\right) \mathrm{d} \lambda}{\left(1-d_{1}\right) \bar{\sigma}}, \\ \mathrm{d} \varepsilon_{22}^{\mathrm{p}}=\mathrm{d} \lambda \frac{\partial f}{\partial \sigma_{22}}=\frac{\mu F\left(\tilde{\sigma}_{11}+\mu \tilde{\sigma}_{22}\right) \mathrm{d} \lambda}{\left(1-d_{2}\right) \bar{\sigma}}, \\ \mathrm{d} \varepsilon_{12}^{\mathrm{p}}=\mathrm{d} \lambda \frac{\partial f}{\partial \sigma_{12}}=\frac{2 \tilde{\sigma}_{12} \mathrm{~d} \lambda}{\left(1-d_{6}\right) \bar{\sigma}} .\end{array}\right.$

From the equations above $F$ and $\mu$ under uniaxial tensile can be expressed by

$\left\{\begin{array}{l}\mu=\frac{\left(1-d_{2}\right) \mathrm{d} \varepsilon_{22}^{\mathrm{p}}}{\left(1-d_{1}\right) \mathrm{d} \varepsilon_{11}^{\mathrm{p}}}, \\ F=\frac{2 \tilde{\sigma}_{12}\left(1-d_{1}\right) \mathrm{d} \varepsilon_{11}^{\mathrm{p}}}{\left(\tilde{\sigma}_{11}+\mu \tilde{\sigma}_{22}\right)\left(1-d_{6}\right) \mathrm{d} \varepsilon_{12}^{\mathrm{p}}} .\end{array}\right.$

Obviously, $F$ and $\mu$ from equations above vary with specimen orientation. Fig. 7 illustrates variations of $F$ and $\mu$ depending on the axial strain for various orientations, from cyclic damage tests with off-axis angle of $30^{\circ}$ and $45^{\circ}$. Results in the figure show that $F$ and $\mu$ depends on both orientation and load, however, reach stable values for strain larger than $0.7 \%$. This observation implies that both $F$ and $\mu$ can be approximated by the stable values, $F=3.25$ and $\mu=0.5$. As the unstable phase is restricted in a narrow range, the errors induced by the assumption is considered to be negligible.

\subsubsection{Determination of the hardening rule}

In the present work, the experimental results of cyclic tests with offaxis angles of $30^{\circ}, 45^{\circ}, 90^{\circ}$ as well as Iosipescu monotonic damage tests are utilized to determine the hardening rules. For the uniaxial off-axis loading tests the stress in the principal material coordinate can be expressed as

$\left\{\begin{array}{l}\sigma_{11}=\sigma_{\theta} \cos ^{2} \theta, \\ \sigma_{22}=\sigma_{\theta} \sin ^{2} \theta \\ \sigma_{12}=-\sigma_{\theta} \cos \theta \sin \theta .\end{array}\right.$

Substituting Eq. (32) into Eq. (16), the relationship between axial stress $\sigma_{\theta}$ and equivalent stress $\bar{\sigma}$ can be written as

$\bar{\sigma}=\sigma_{\theta} h_{\theta}$

with

$h_{\theta}=\sqrt{F\left(\frac{\cos ^{2} \theta}{1-d_{1}}+\frac{\sin ^{2} \theta}{1-d_{2}}\right)^{2}+\frac{2 \sin ^{2} \cos ^{2} \theta}{\left(1-d_{6}\right)^{2}}}$.

According to the strain transformation, the plastic part of the axial strain becomes

$\mathrm{d} \varepsilon_{\theta}^{\mathrm{p}}=\mathrm{d} \varepsilon_{11}^{\mathrm{p}} \cos ^{2} \theta+\mathrm{d} \varepsilon_{22}^{\mathrm{p}} \sin ^{2} \theta-\mathrm{d} \varepsilon_{12}^{\mathrm{p}} \cos \theta \sin \theta$.

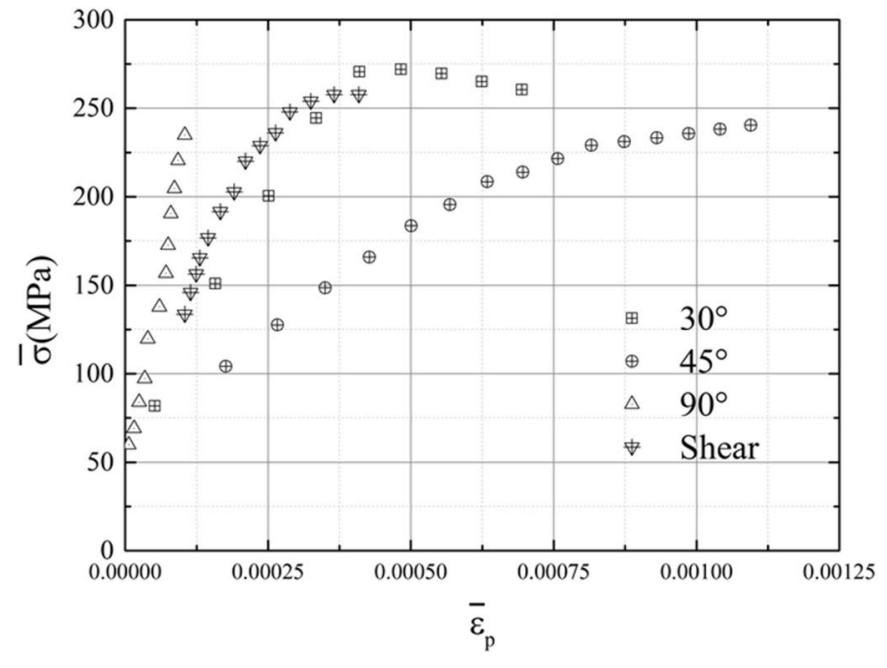

Fig. 8. Hardening curves.

Substituting the plastic strain increment in Eq. (30) into Eq. (35) and combining with Eq. (18), the relationship between the incremental axial plastic strain and equivalent plastic strain is obtained, as

$\mathrm{d} \bar{\varepsilon}^{\mathrm{p}}=\mathrm{d} \varepsilon_{\theta}^{\mathrm{p}} / h_{\theta}$.

Based on Eq. (33) and Eq. (36), the $\bar{\sigma}-\bar{\varepsilon}^{\mathrm{p}}$ curves for uniaxial loading tests can be transformed from experimental curves and plotted in Fig. 8.

In the Iosipescu monotonic shear damage test, $\tilde{\sigma}_{11}$ and $\tilde{\sigma}_{22}$ equal zero, the expression of the equivalent stress is reduced to $\bar{\sigma}=\sqrt{2} \tilde{\sigma}_{12}$. The expression of equivalent plastic strain increment is simplified into $\mathrm{d} \bar{\varepsilon}^{\mathrm{p}}=\varepsilon_{12}^{\mathrm{p}} / \sqrt{2}$. by using Eq. (20). The exponents $n$ and $t$ in Eq. (20) are determined to be 1.3. The coefficients $A$ and $m$ depend on loading orientation and have to be identified from off-axis tension tests. The results are summarized on Fig. 9. In figure $A$ and $m$ are expressed by the dimensionless loading parameter $\omega$, which can be approximated by polynomial functions as

$A=A_{0}+A_{1} \omega+A_{2} \omega^{2}, m=m_{0}+m_{1} \omega+m_{2} \omega^{2}$

with

$A_{0}=5.93213 \times 10^{7}, A_{1}=-2.51889 \times 10^{8}, A_{2}=2.71052 \times 10^{8}$,

$m_{0}=9.41509 \times 10^{4}, m_{1}=-4.08725 \times 10^{5}, m_{2}=4.47389 \times 10^{5}$.

\subsubsection{Model parameters for monotonic damage evolution}

The parameters in the monotonic damage evolution are determined based on the results of monotonic damage tests. In Eq. (27), the shear

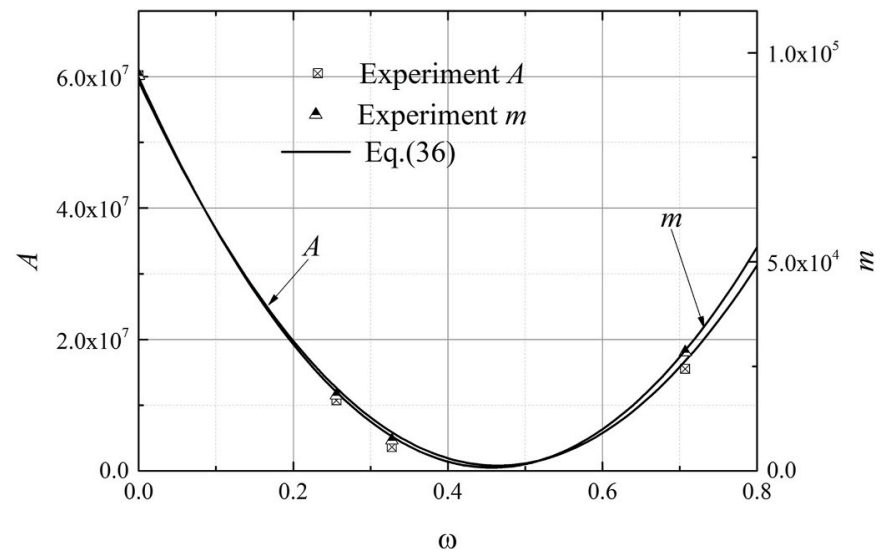

Fig. 9. Variations of the parameters $A$ and $m$ in the hardening function for different loading orientations. Legend Experiments; Eq. (36). 


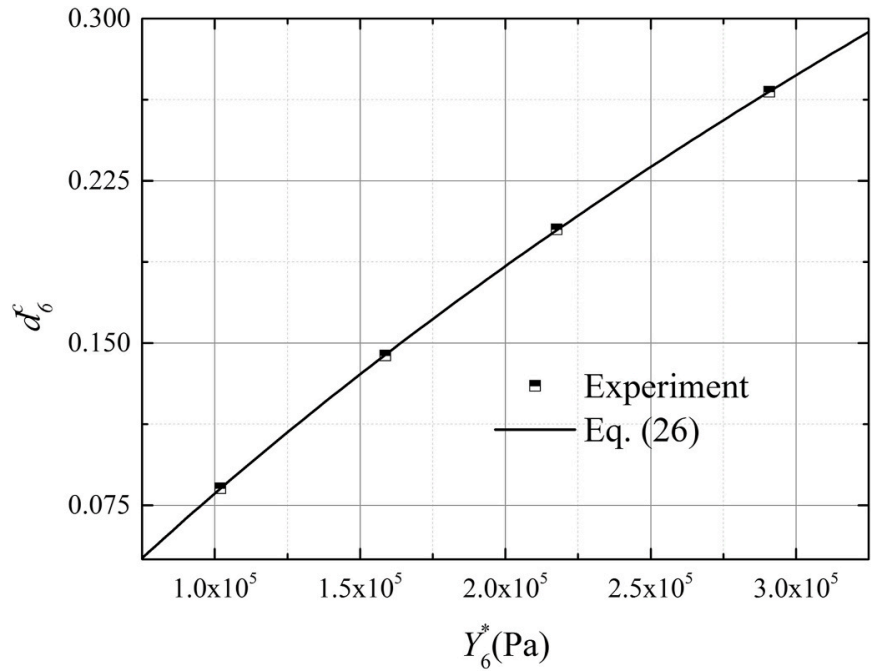

Fig. 10. The monotonic damage evolution in the Iosipescu shear test as a function of the thermodynamic force. legend: Experiments; Eq. (26).

damage $d_{6}$ is a function of $Y_{6}^{*}$. In Iosipescu shear tests, only the stress component $\sigma_{12}$ exists, i.e. $Y_{6}^{*}=Y_{6}$. The parameters for the shear damage evolution can be identified from fitting the curve of $d_{6}-Y_{6}$ for Iosipescu shear tests, the relationship between $d_{6}$ and $Y_{6}$ plotted in Fig. 10.

After the Iosipescu shear test, the expression of $Y_{6}^{*}$ is determined by the experimental data of $45^{\circ}$ off-axis tension tests. $Y_{6}^{*}$ can be further determined as $a_{61}=-1.10788, a_{62}=-0.43746$ and $a_{66}=1$. So far the evolution of monotonic shear damage $d_{6}^{m}$ is determined.

It is known that the composites display very slight damage in the materials principal directions and are damaged mainly by shearing. For this reason it is assumed in the present work that $Y_{6}$ plays a much more significant role in the damage evolution than $Y_{1}$ and $Y_{2}$. Based on this postulate, the equivalent energy release rates $Y_{1}^{*}$ and $Y_{2}^{*}$ are dominated by $Y_{6}$ and can be approximately expressed as

$Y_{1}^{*} \approx Y_{6}, \quad Y_{2}^{*} \approx Y_{6}$.

Then the evolution equations of $d_{1}^{m}$ and $d_{2}^{m}$ can be determined based on the experimental data of $45^{\circ}$ off-axis loading tests, the fitting results of $d_{1}^{m}$ and $d_{2}^{m}$ are plotted in Fig. 11. The present damage evolution is dominated by the shear strain energy.

In the damage evolution equation, Eq. (27) three additional model parameters are introduced, which are summarized in Table.1.

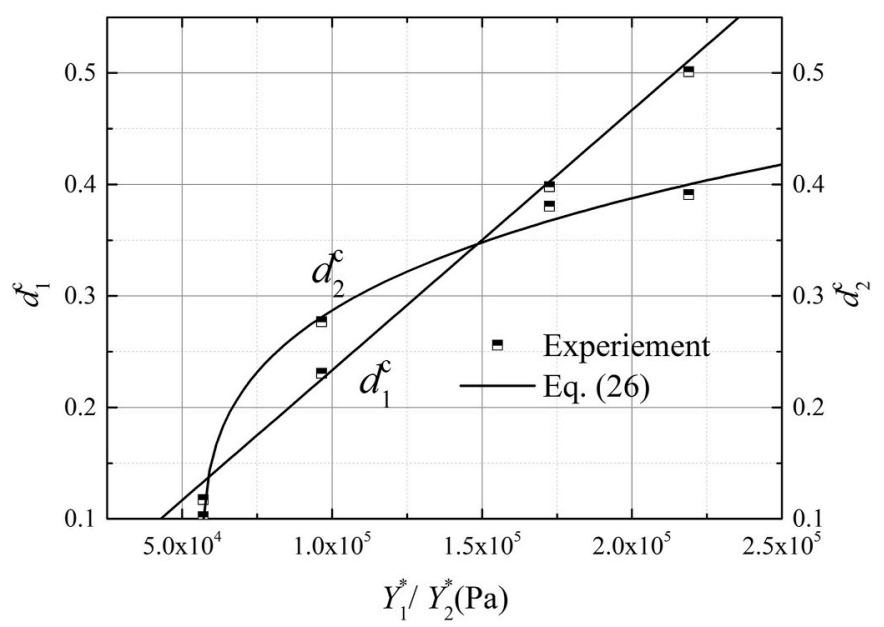

Fig. 11. The monotonic damage evolution in the fiber directions versus to thermodynamic forces. LegendExperiments Eq. (26).
Table 1

Parameters for the monotonic damage evolution model.

\begin{tabular}{llll}
\hline$i$ & 1 & 2 & 6 \\
\hline$\gamma_{i}$ & $4.17198 \times 10^{-5}$ & $1.93601 \times 10^{-2}$ & $1.86325 \times 10^{-5}$ \\
$Y_{i}^{0}$ & $2.72784 \times 10^{4}$ & $5.63123 \times 10^{4}$ & $4.88119 \times 10^{4}$ \\
$t_{i}$ & 0.77185 & 0.25236 & 0.77179 \\
\hline
\end{tabular}

\subsubsection{Model parameters for cyclic damage evolution}

In determination of the monotonic damage evolution model, cyclic damage was not considered. The cyclic damage $d_{i}^{c}$ defined in Eq. (12) represents as the difference between the total damage and the monotonic damage. The experimental results of cyclic loading tests in $30^{\circ}$ and $45^{\circ}$ are adopted to determine the model parameters for cyclic damage evolution.

As increments of cyclic damage in each unloading-reloading cycle is tiny compared to monotonic damage. All variables within a cycle can be approximated by linear interpolations. The damage evolution equation in Eq. (29) can simplified into

$\Delta d_{i}^{c}=\zeta_{i} \frac{\bar{\sigma}_{\max }}{\bar{\varepsilon}_{\max }^{p}} d_{i}^{\alpha_{i}} \Delta W(i=1,2,6)$,

where $\Delta d_{i}^{c}$ and $\Delta W$ represent the increments of cyclic damage and strain energy in a loading cycle, which can be calculated from stressstrain curves measured in experiments. To identify the model parameters, Eq. (39) can be rewritten as

$\zeta_{i}\left(d_{i}^{c}\right)^{\alpha_{i}}=\frac{\bar{\varepsilon}_{\max }^{p}}{\bar{\sigma}_{\max }} \frac{\Delta d_{i}}{\Delta W}=\delta_{i}$,

where $\delta_{i}$ is introduced for determining the model parameters. Obviously $\delta_{i}$ can be calculated from experimental results. Using the least square method one determines the model parameters $\zeta_{i}$ and $\alpha_{i}$. Correlations between $\delta_{i}$ and $d_{i}$ are illustrated in Fig. 12 for comparison between the experimental and fitting results. Values of $\zeta_{i}$ and $\alpha_{i}$ are listed in Table 2.

\section{Verifications and discussions}

The model was implemented into the commercial finite element code ABAQUS UMAT and validated by predicting the stress-strain curves for different loading conditions including monotonic tensile tests and cyclic loading-unloading tests with various off-axis angles. An set of additional experiments for monotonic and cyclic loading with off-axis angle $60^{\circ}$ were also conducted to verify the models introduced in the present work. The comparison between computations and experiments is shown in Fig. 13 for monotonic loading and Fig. 14 for cyclic loadings. In Fig. 14, one representative loop of every five cycles is selected to plot for off-axis cyclic tests $\left(30 / 45 / 60^{\circ}\right)$ and only initial and last loops are plotted for the on-axis cyclic tests $\left(0 / 90^{\circ}\right)$ :

As discussed in the previous sections, the model parameters for plasticity were determined from stress-strain curves without significant material degradation. The monotonic damage model parameters were identified from the monotonic tests, beyond the plastic regions. As soon as $E$ changes, the material is damaging and the monotonic damage evolution begins. The parameters for the cyclic damage were determined from the cyclic loading tests, where both monotonic damage and cyclic damage exist. The cyclic damage is the additional material degradation just due to cyclic loading process. The computational results confirm good agreement to experiments. Both plastic stress-strain envelop as well as reduction process of $E$ provide reasonable approaches to the experiments. The relative errors of the plastic strain accumulation were obviously larger than those of the damage evolution, which is perhaps from the assumption of the constant parameters in plastic potential function. 


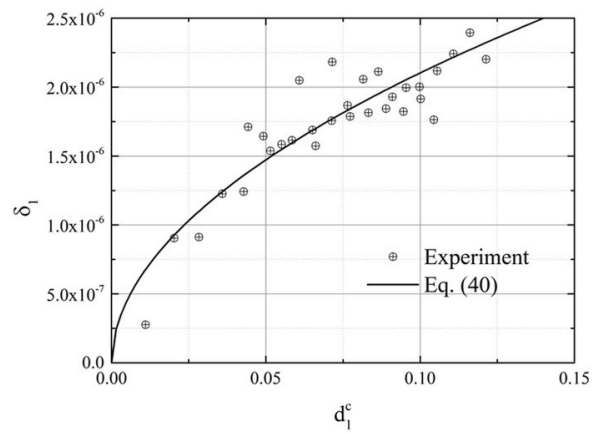

(a)

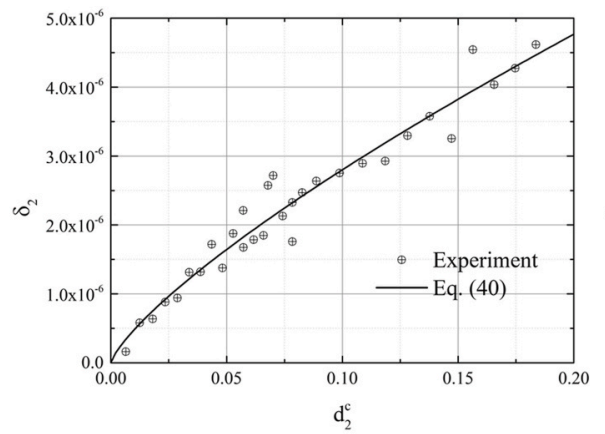

(b)

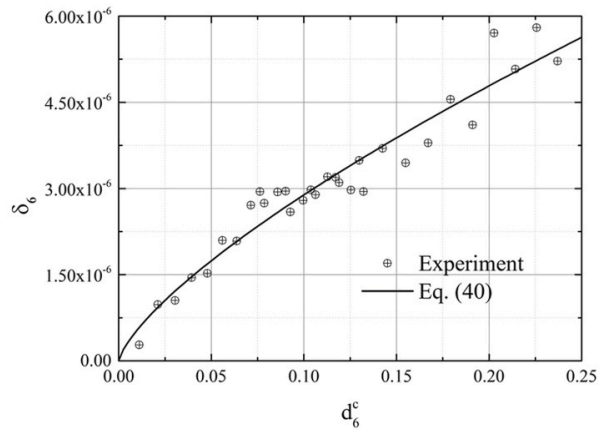

(c)

Fig. 12. The relationship between parameter $\delta_{i}$ and cyclic damage. $d_{i}^{c}$

Table 2

Parameters in the cyclic damage evolution model.

\begin{tabular}{llll}
\hline$i$ & 1 & 2 & 6 \\
\hline$\zeta_{i}$ & $6.869 \times 10^{-6}$ & $1.641 \times 10^{-5}$ & $1.546 \times 10^{-5}$ \\
$\alpha_{i}$ & 0.5142 & 0.7679 & 0.7287 \\
\hline
\end{tabular}

\section{Conclusions}

Three sets of comparative experiments showed that the multiple loading-unloading cycles would have significant influence on the mechanical behavior of oxide/oxide ceramic matrix composites, including the stress-strain response and the stiffness degradations. Higher damage accumulation rates would induce by the loading-unloading process. This observation implies the necessity to introduce damage into continuum mechanics model for the composites.

The composites can be damaged in a fatigue process. Based on the assumption that the damage can be decomposed into monotonic damage and cyclic damage, a new constitutive model for multiaxial elastic-plastic damage is proposed in the present work. The model reveals that the composites are damaged mainly by shearing represented by the shear strain energy density. Comparison between the experimental results and computations, the model is proved to be efficient to predict the damage evolution and nonlinear mechanical behaviors in monotonic and cyclic loading conditions.

\section{Declaration of competing interest}

The authors declare that they have no known competing financial interests or personal relationships that could have appeared to influence the work reported in this paper.

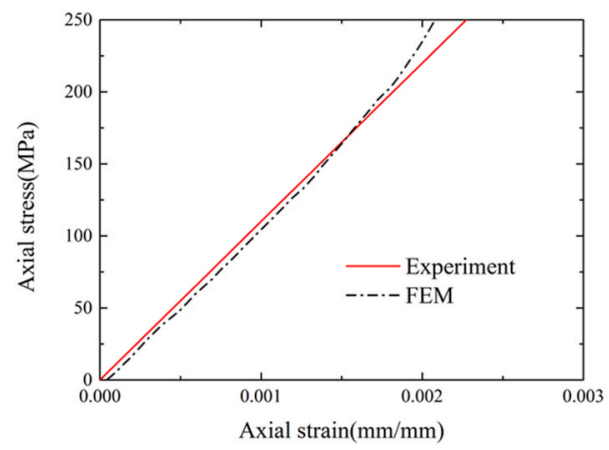

(a) $0^{\circ}$

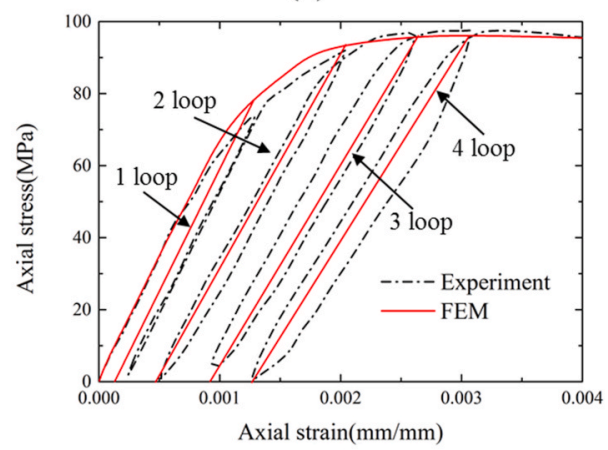

(d) $60^{\circ}$

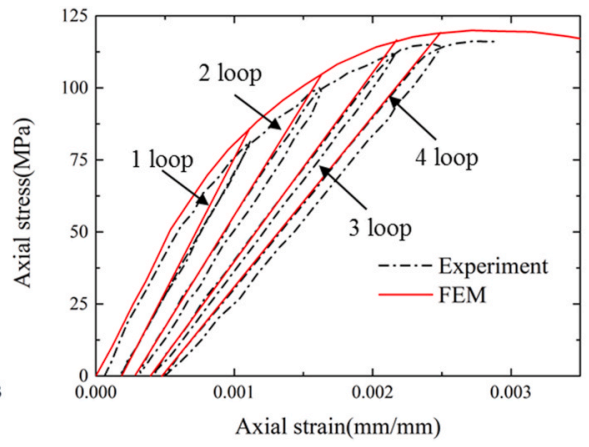

(b) $30^{\circ}$

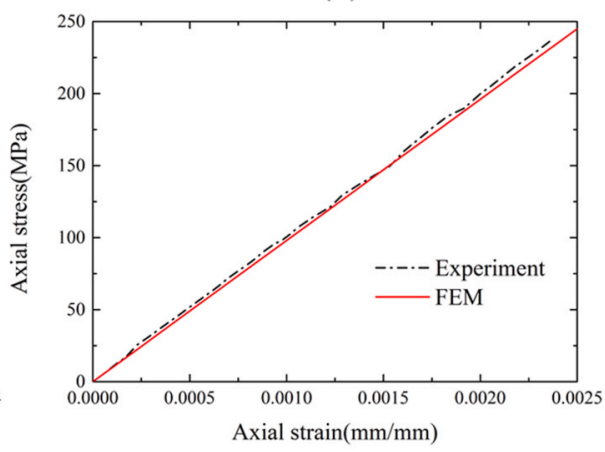

(e) $90^{\circ}$

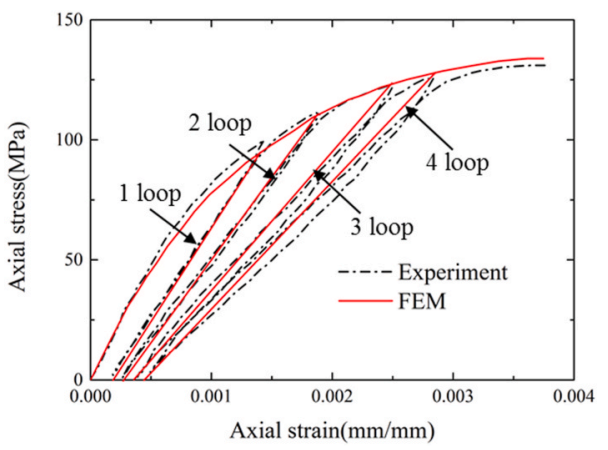

(c) $45^{\circ}$

Fig. 13. The comparison between experimental results and FEM analysis under monotonic loading conditions. 


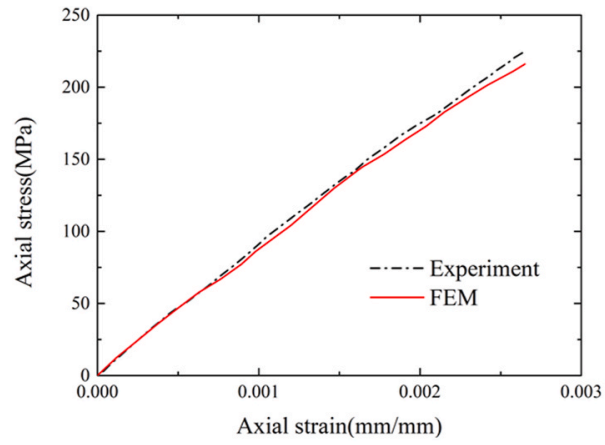

(a) $0^{\circ}$

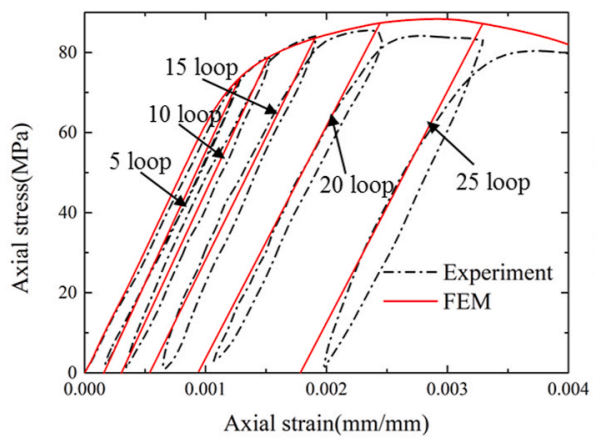

(d) $60^{\circ}$

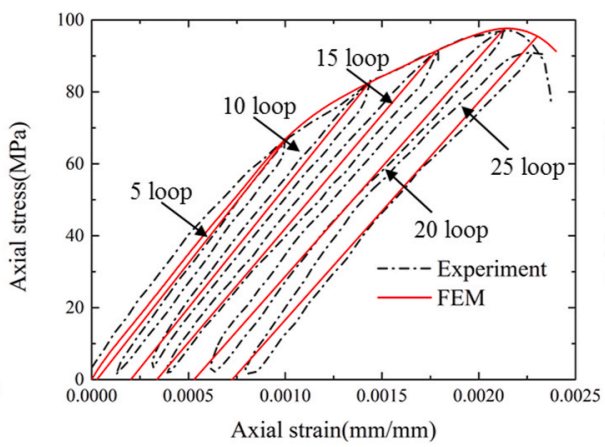

(b) $30^{\circ}$

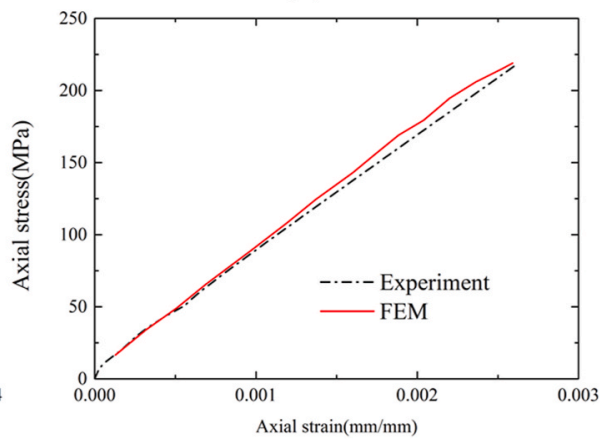

(e) $90^{\circ}$

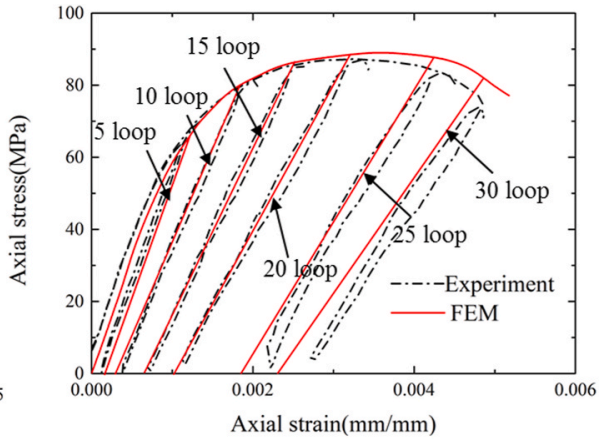

(c) $45^{\circ}$

Fig. 14. The comparison between experimental results and FEM analysis in cyclic loading conditions.

\section{Appendix A. Supplementary data}

Supplementary data to this article can be found online at https:// doi.org/10.1016/j.ceramint.2020.05.208.

\section{References}

[1] H. Kaya, The application of ceramic-matrix composites to the automotive ceramic gas turbine, Compos. Sci. Technol. 59 (6) (1999) 861-872.

[2] R. Knoche, E. Werth, M. Weth, J. G. García, C. Wilhelmi, M. Gerendás, Design and development approach for gas turbine combustion chambers made of oxide ceramic matrix composites, Mechanical Properties and Performance of Engineering Ceramics and Composites VI: Ceram. Eng. Sci. Proc. 32.

[3] S. Tang, C. Hu, Design, preparation and properties of carbon fiber reinforced ultrahigh temperature ceramic composites for aerospace applications: a review, J. Mater. Sci. Technol. 33 (2) (2017) 117-130.

[4] E. Volkmann, K. Tushtev, D. Koch, C. Wilhelmi, J. Göring, K. Rezwan, Assessment of three oxide/oxide ceramic matrix composites: mechanical performance and effects of heat treatments, Compos. Appl. Sci. Manuf. 68 (2015) 19-28.

[5] G. Blugan, C. Strehler, M. Vetterli, B. Ehrle, R. Duttlinger, P. Blösch, J. Kuebler, Performance of lightweight coated oxide ceramic composites for industrial high speed wood cutting tools: a step closer to market, Ceram. Int. 43 (12) (2017) 8735-8742.

[6] N.P. Bansal, Handbook of Ceramic Composites vol. 200, Springer Science \& Business Media, 2006.

[7] D. Koch, K. Tushtev, G. Grathwohl, Ceramic fiber composites: experimental analysis and modeling of mechanical properties, Compos. Sci. Technol. 68 (5) (2008) 1165-1172 (recent Advances in Experimental and Applied Research of Composite Materials).

[8] C.B. Ramdane, A. Julian-Jankowiak, R. Valle, Y. Renollet, M. Parlier, E. Martin, P. Diss, Microstructure and mechanical behaviour of a Nextel"610/alumina weak matrix composite subjected to tensile and compressive loadings, J. Eur. Ceram. Soc. 37 (8) (2017) 2919-2932.

[9] S. Baste, Inelastic behaviour of ceramic-matrix composites, Compos. Sci. Technol. 61 (15) (2001) 2285-2297.

[10] T. Whitlow, E. Jones, C. Przybyla, In-situ damage monitoring of a sic/sic ceramic matrix composite using acoustic emission and digital image correlation, Compos. Struct. 158 (2016) 245-251.

[11] V. Mazars, O. Caty, G. Couégnat, A. Bouterf, S. Roux, S. Denneulin, J. Pailhès, G.L. Vignoles, Damage investigation and modeling of 3d woven ceramic matrix composites from x-ray tomography in-situ tensile tests, Acta Mater. 140 (2017) $130-139$.

[12] S. Mukhopadhyay, S.R. Hallett, A directed continuum damage mechanics method for modelling composite matrix cracks, Compos. Sci. Technol. 176 (2019) 1-8.
[13] K.L. Reifsnider, V. Tamuzs, S. Ogihara, On nonlinear behavior in brittle heterogeneous materials, Compos. Sci. Technol. 66 (14) (2006) 2473-2478 (special Issue in Honour of Professor C.T. Sun).

[14] A. Doitrand, C. Fagiano, V. Chiaruttini, F. Leroy, A. Mavel, M. Hirsekorn, Experimental characterization and numerical modeling of damage at the mesoscopic scale of woven polymer matrix composites under quasi-static tensile loading, Compos. Sci. Technol. 119 (2015) 1-11.

[15] U. Santhosh, J. Ahmad, G. Ojard, R. Miller, Y. Gowayed, Deformation and damage modeling of ceramic matrix composites under multiaxial stresses, Compos. B Eng. 90 (2016) 97-106.

[16] ingran Ge, C. He, J. Liang, Y. Chen, D. Fang, A coupled elastic-plastic damage model for the mechanical behavior of three-dimensional (3d) braided composites, Compos. Sci. Technol. 157 (2018) 86-98.

[17] H. Choi, C. Heinrich, W. Ji, An efficient homogenization technique for fiber tows in textile composites with emphasis on directionally dependent nonlinear stress-strain behavior, Compos. Struct. 208 (2019) 816-825.

[18] C. He, J. Ge, D. Qi, J. Gao, Y. Chen, J. Liang, D. Fang, A multiscale elasto-plastic damage model for the nonlinear behavior of $3 \mathrm{~d}$ braided composites, Compos. Sci. Technol. 171 (2019) 21-33.

[19] V.P. Rajan, J.H. Shaw, M.N. Rossol, F.W. Zok, An elastic-plastic constitutive model for ceramic composite laminates, Compos. Appl. Sci. Manuf. 66 (2014) 44-57.

[20] U. Mandel, R. Taubert, R. Hinterhölzl, Three-dimensional nonlinear constitutive model for composites, Compos. Struct. 142 (2016) 78-86.

[21] G. Abu-Farsakh, A. Asfa, Macro-mechanical damage modeling of fibrous composite materials accounting for non-linear material behavior, Compos. Sci. Technol. 156 (2018) 287-295.

[22] S. Zhong, L. Guo, G. liu, H. Lu, T. Zeng, A continuum damage model for threedimensional woven composites and finite element implementation, Compos. Struct. 128 (2015) 1-9.

[23] H. Guo, B. Wang, P. Jia, C. Yang, In-plane shear behaviours of a 2d-sic/sic composite under various loading conditions, Ceram. Int. 41 (9, Part A) (2015) 11562-11569.

[24] C. Hochard, Y. Thollon, A generalized damage model for woven ply laminates under static and fatigue loading conditions, Int. J. Fatig. 32 (1) (2010) 158-165.

[25] Y. Thollon, C. Hochard, A general damage model for woven fabric composite laminates up to first failure, Mech. Mater. 41 (7) (2009) 820-827.

[26] C. Sun, J. Chen, A simple flow rule for characterizing nonlinear behavior of fiber composites, J. Compos. Mater. 23 (10) (1989) 1009-1020.

[27] S. Ogihara, S. Kobayashi, K.L. Reifsnider, Characterization of nonlinear behavior of carbon/epoxy unidirectional and angle-ply laminates, Adv. Compos. Mater. 11 (3) (2002) 239-254.

[28] K. Ogi, N. Takeda, Effects of moisture content on nonlinear deformation behavior of cf/epoxy composites, J. Compos. Mater. 31 (6) (1997) 530-551.

[29] J. Xie, G. Fang, Z. Chen, J. Liang, An anisotropic elastoplastic damage constitutive model for 3d needled c/c-sic composites, Compos. Struct. 176 (2017) 164-177.

[30] T. Flatscher, H. Pettermann, A constitutive model for fiber-reinforced polymer plies accounting for plasticity and brittle damage including softening - implementation 
for implicit fem, Compos. Struct. 93 (9) (2011) 2241-2249.

[31] G. Vyas, S. Pinho, P. Robinson, Constitutive modelling of fibre-reinforced composites with unidirectional plies using a plasticity-based approach, Compos. Sci. Technol. 71 (8) (2011) 1068-1074.

[32] Z. Yang, H. Yuan, H. Liu, Evolution and characterization of cyclic thermal shockinduced thermomechanical damage in oxide/oxide ceramics matrix composites, Int. J. Fatig. 120 (2019) 150-161.

[33] Z. Yang, H. Yuan, B. Markert, Representation of micro-structural evolution and thermo-mechanical damage in thermal shocked 2-D woven oxide/oxide ceramic matrix composites, Int. J. Fatig. 126 (2019) 122-129.

[34] Z. Yang, H. Liu, H. Yuan, Micro-porosity as damage indicator for characterizing cyclic thermal shock-induced anisotropic damage in oxide/oxide ceramic matrix composites, Eng. Fract. Mech. 220 (2019) 106669.

[35] J. Lemaitre, R. Desmorat, Engineering Damage Mechanics: Ductile, Creep, Fatigue and Brittle Failures, Springer-Verlag Berlin Heidelberg, 2005.

[36] H. Yuan, S. Ma, L. Zhang, Continuum damage mechanics for sintered powder metals, Sci. China Phys. Mech. Astron. 58 (2015) 1-12.

[37] S. Ma, B. Markert, H. Yuan, Multiaxial fatigue life assessment of sintered porous iron under proportional and non-proportional loadings, Int. J. Fatig. 97 (2017) 214-226.

[38] S. Ma, H. Yuan, A continuum damage model for multi-axial low cycle fatigue of porous sintered metals based on the critical plane concept, Mech. Mater. 104 (2017) 13-25.

[39] Astm-D3518, Standard Test Method for In-Plane Shear Response of Polymer Matrix Composite Materials by Tensile Test of a $\pm 45^{\circ}$ Laminate, American Society for Testing and Materials D3518/D3518M.

[40] G.Z. Voyiadjis, P.I. Kattan, Damage Mechanics, CRC Press, Boca Raton, 2005.

[41] H. Liu, Z. Yang, H. Yuan, A novel elastoplastic constitutive model for woven oxide/ oxide ceramic matrix composites with anisotropic hardening, Compos. Struct. 229 (2019) 111420

[42] Y.-y. Jiang, A fatigue criterion for general multiaxial loading, Fatig. Fract. Eng. Mater. Struct. 23 (1) (2000) 19-32. 